\title{
Mean field effects in heavy-ion collisions at AGS energies
}

\author{
Bao-An Li, C. M. Ko, and G. Q. Li \\ Cyclotron Institute and Physics Department, \\ Texas A $6 M$ University, College Station, TX 77843
}

The question whether mean field effects exist in heavy-ion collisions at AGS energies is studied in the framework of A Relativistic Transport (ART) model. It is found that in central collisions of $\mathrm{Au}+\mathrm{Au}$ at $P_{\text {beam }} / A=11.6 \mathrm{GeV} / \mathrm{c}$ a simple, Skyrme-type nuclear mean field satisfying the causality requirement reduces the maximum baryon and energy densities reached in the cascade model by about $30 \%$ and $40 \%$, respectively. The mean field affects the inclusive, single particle observables of various hadrons by at most $20 \%$. We show, however, that the mean field causes a factor of 2.5 increase in the strength of the baryon transverse collective flow. 
The purpose of relativistic heavy-ion collisions at AGS energies is to study the properties of hot and dense hadronic matter and the possible phase transition to the quark-gluon plasma. A vast body of data have been collected and analysed during the past years [1]. Comparisons of these data with theoretical models, such as RQMD [2], ARC [3] and QGSM [4], have revealed much interesting physics. In particular, a picture of nearly complete stopping of baryons in central heavy-ion collisions at AGS energies has emerged from these studies. It has been shown that baryon and energy densities up to 10 times that of nuclei in their ground state have been reached in these collisions. This has led to the suggestion that the energy and baryon densities achieved during central heavy-ion collisions at AGS energies may be high enough to form the quark-gluon plasma [5]. However, the above suggestion and many speculations critically rely on the inferred maximum energy and baryon densities reached in the reaction.

In this Letter, we report on the main aspects of a relativistic transport (ART) model for heavy-ion collisions at AGS energies, and study, in particular, effects of the nuclear mean field which has been ignored in ARC. Our motivations of studying the nuclear mean field effects are mainly the following. Firstly, we believe that the mean-field potential is not negligible in heavy-ion collisions at AGS energies. Although the forward scattering amplitudes of hadronhadron collisions in the high energy limit have been found approximately purely imaginary [6], the AGS energies may not be high enough for the real part of the scattering amplitude to completely vanish. Of course, the form and strength of the corresponding mean field in the hot and dense hadronic matter is highly uncertain and has been a subject of much discussions. Secondly, although the kinetic energy is much higher than the potential energy in the early stage of the reaction, particles are gradually slowed down and the mean field plays an increasingly important role as the reaction goes on. In particular, the repulsive mean field in the high density region tends to keep particles from coming too close to each other and therefore reduce the maximum energy and baryon densities reached in the reaction should there be no mean field. Moreover, in the expansion phase of the reaction mean-field effects are expected to be even stronger. It is therefore necessary to study how 
the compression and expansion are affected by the nuclear mean field. Finally, what are the experimentally observable consequences of the nuclear mean field ?

Our relativistic transport model is developed from the well known Boltzmann-UhlingUehlingbeck (BUU) model (e.g. [7,8]) for intermediate energy heavy-ion collisions. Here we report on the extension of the model to AGS energies with a reasonable amount of details. We have included in the model the following baryons: $N, \Delta(1232), N^{*}(1440), N^{*}(1535), \Lambda, \Sigma$, and mesons: $\pi, \rho, \omega, \eta, K$, with their explicit isospin degrees of freedom. Both elastic and inelastic collisions among most of these particles are simulated as best as we can using as much input from the experimental hadron-hadron data as possible [9]. Most of the inelastic hadron-hadron collisions are modeled through the formation of resonances. The finite lifetimes of these resonances effectively take into account the formation time of newly produced secondaries. More specifically, we have included in the model

$$
\begin{aligned}
& N N \leftrightarrow N \Delta, N N^{*}(1440), N N^{*}(1535), \\
& N N \leftrightarrow \Delta \Delta, \Delta N^{*}(1440), \\
& N N \rightarrow N N \rho, N N \omega, \Delta \Delta \pi, \\
& N N \rightarrow \Delta \Delta \rho, \\
& N \Delta \leftrightarrow N N^{*}(1440), N N^{*}(1535), \\
& \Delta \Delta \leftrightarrow N N^{*}(1440), N N^{*}(1535), \\
& \Delta N^{*}(1440) \leftrightarrow N N^{*}(1535),
\end{aligned}
$$

and those producing kaons

$$
\begin{aligned}
& N N \rightarrow N \Lambda(\Sigma) K, \Delta \Lambda(\Sigma) K \\
& N R \rightarrow N \Lambda(\Sigma) K, \Delta \Lambda(\Sigma) K \\
& R R \rightarrow N \Lambda(\Sigma) K, \Delta \Lambda(\Sigma) K
\end{aligned}
$$

where $\mathrm{R}$ is $\Delta, N^{*}(1440)$ or $N^{*}(1535)$. Cross sections parameterized by VerWest et al. [10] are used for the single $\Delta$ and $N^{*}(1440)$ production in processes (1). The cross section of 
$N^{*}(1535)$ production is estimated from that of $\eta$ production [11]. Cross sections for the double resonances production in processes (2) are taken to be the same for all possible isospin channels, this is supported by recent calculations based on the one-boson-exchange model [12]. Numerically, it is estimated by subtracting from the inclusive $2 \pi$ production cross section [9] the contribution of $N N \rightarrow N N \rho$ and the $2 \pi$ decay of the $N^{*}(1440)$ in the $N N \rightarrow N N^{*}(1440)$ process. The cross sections for $\rho$ and $\omega$ production in channels (3) are taken directly from the experimental data [9]. The cross section for the process $N N \rightarrow \Delta \Delta \pi$ is taken as the difference between the inclusive $3 \pi$ and the $\omega$ production cross section. We attribute the difference between the experimental total nucleon-nucleon inelastic cross section and the sum of cross sections for channels (1) to (3) as well as the kaon production cross sections of channels (8) to the process $N N \rightarrow \Delta \Delta \rho$. This is done to ensure the total inelasticity of nucleon-nucleon collisions as only a limited, though large, number of reaction channels have been incorporated so far. The bias introduced by this approximation towards the cross section of the quasi- $4 \pi$ production process $N N \rightarrow \Delta \Delta \rho$ is very small. Cross sections for channels (5) to (7), (9) and (10) are taken to be the same as in nucleon-nucleon collisions having the same center of mass energy and total charge. Cross sections for the inverse processes are calculated by using the detailed balance. Masses of baryon and meson resonances are generated according to the single or joint Breit-Wigner distributions with momentum dependent widths using the rejection method.

One can also separate meson-baryon collisions into elastic and inelastic parts. We model elastic collisions through both the formation of baryon resonances, i.e., $\pi N \leftrightarrow$ $\Delta\left(N^{*}(1440), N^{*}(1535)\right)$ and $\eta N \leftrightarrow N^{*}(1535)$, as well as direct processes, i.e., $\pi(\rho)+$ $N\left(\Delta, N^{*}\right) \rightarrow \pi(\rho)+N\left(\Delta, N^{*}\right)$, and $K+N \rightarrow K+N$. The formation of the three baryon resonances accounts almost completely the $\pi+N$ elastic cross sections at low energies. At higher energies, for $\pi^{-}+p$ at $\sqrt{s} \geq 1.7 \mathrm{GeV}$, for example, the elastic cross section is about $7 \mathrm{mb}$ and is mainly due to the formation of higher resonances which are not included in the present model. We therefore attribute the difference between the experimental elastic cross section and the contribution from the three baryon resonances to the direct process 
$\pi+N \rightarrow \pi+N$. For experimentally unknown cross sections, such as, $\pi^{0}+N, \pi+\Delta\left(N^{*}\right)$ and $\rho+N$, we calculate them using the resonance model. Neglecting the interference between resonances, one has

$$
\sigma(M+B)=1.3 \frac{\pi}{k^{2}} \sum_{R} \frac{\left(2 J_{R}+1\right)}{\left(2 S_{M}+1\right)\left(2 S_{B}+1\right)} \frac{\Gamma_{R}^{2}(M+B)}{\left(\sqrt{s}-m_{R}\right)^{2}+0.25 \Gamma_{R}^{2}(\text { total })} .
$$

The factor 1.3 is obtained by fitting to the high energy part of the $\pi^{+}+p$ data. For calculating the cross sections of direct channels, $\mathrm{R}$ runs over all baryon resonances with masses up to 2 $\mathrm{GeV}$.

The $\pi+N$ inelastic collision mainly goes through the production of pions and kaons. Similar to the treatment of baryon-baryon collisions, we model the inelastic $\pi+N$ collisions through the production of resonances, namely, $\pi+N \leftrightarrow \Delta+\pi(\rho, \omega)$ and $\pi(\rho, \omega)+N\left(\Delta, N^{*}\right) \rightarrow \Lambda(\Sigma)+K$. All experimental cross sections for $\pi+N$ collisions with final states having two and three pions are attributed to the production of $\Delta \pi$ and $\Delta \rho$, respectively. The difference between the experimental total $\pi+N$ inelastic cross section and cross sections for the production of two and three pions as well as kaons are attributed to the production of $\Delta \omega$. Cross sections for the production of kaons in $\pi(\rho, \omega)+N\left(\Delta, N^{*}\right)$ collisions are taken to be the same as that in the $\pi+N$ collision.

We model pion-pion elastic collisions through the formation of the $\rho$ meson and the direct process. The latter takes into account the case when the quantum numbers of colliding pions forbid the formation of the $\rho$ meson. These cross sections are taken mainly from ref. [15]. The inelastic collisions among mesons are modeled through the production of $K \bar{K}$. The cross section for this process is highly uncertain, here we use the cross section calculated from the $K^{*}$-exchange model of ref. [16].

The "leading" particle behaviour in energetic collisions is ensured by requiring the outgoing baryons to have the same or similar quantum numbers as the incident ones so that their longitudinal directions are retained when performing the momentum transformation from the baryon-baryon c.m. frame to the nucleus-nucleus c.m. frame. The longitudinal and transverse momenta of baryons in the final state is assigned according to the following 
distribution under the constraint of total energy and momentum conservations [13

$$
\frac{d^{2} N}{d^{3} \overrightarrow{p^{*}}} \propto\left(1 .+0.5 x^{*}-0.9 x^{*^{2}}\right)\left(e^{-4 p_{t}^{2}}+0.5 e^{-10 p_{t}}\right)
$$

where $x^{*}$ is the scaled longitudinal momentum in the c.m. frame, i.e., $x^{*}=2 p_{z}^{*} / \sqrt{s}$. The distribution has the properties of the naive scaling, $p_{t}$ and $p_{z}^{*}$ factorization and the soft, energy independent transverse momentum distribution. With this distribution and the cross sections discussed above, we can well reproduce the longitudinal and transverse momentum distributions of protons and pions in $p p$ collisions at $p_{\text {beam }}=12 \mathrm{GeV} / \mathrm{c}$ and below. The test against $p p$ data together with many other details of the model will be published separately [14.

In the framework of the ART model outlined above, we shall concentrate on studying the effects of nuclear mean field. Without much reliable knowledge about the nuclear equation of state in hot and dense medium, we use here the simple, Skyrme-type parameterization widely used at Bevalac energies and below,

$$
U(\rho)=-358.1\left(\frac{\rho}{\rho_{0}}\right)+304.8\left(\frac{\rho}{\rho_{0}}\right)^{1.167} .
$$

This is the so-called soft equation of state which satisfies the causality requirement upto about $7 \rho_{0}$. The standard test-particle method is used to calculate the global baryon density $\rho_{g}$ and energy density $e_{g}$ in the nucleus-nucleus c.m. frame on a lattice with the cell size of $1 \mathrm{fm}^{3}$. The local baryon density and energy density in each cell are obtained by $\rho_{l}=\rho_{g} / \gamma$ and $e_{l}=e_{g} / \gamma$, where $\gamma$ is the Lorentz factor in each cell.

Let us first study effects of the mean filed on the creation of high baryon and energy densities. We show in Fig. 1 the evolution of the local baryon and energy densities in the central cell during the reaction of $\mathrm{Au}+\mathrm{Au}$ at $P_{\text {beam }} / A=11.6 \mathrm{GeV} / \mathrm{c}$ and $\mathrm{b}=0$ for three different cases. For the cascade case, it is seen that the maximum baryon density of about $9 \rho_{0}$ and the maximum energy density of about $3.6 \mathrm{GeV} / \mathrm{fm}^{3}$ are reached at about $4 \mathrm{fm} / \mathrm{c}$. The high energy density matter lasts for about $5 \mathrm{fm} / \mathrm{c}$. A more detailed examination of the density contour plots shows that the high energy density region with $e_{l} \geq 2.0 \mathrm{GeV} / \mathrm{fm}^{3}$ has 
the maximum volume of about $200 \mathrm{fm}^{3}$ at about $4 \mathrm{fm} / \mathrm{c}$. It is worth mentioning that the main features of the cascade model calculations are very similar to that of ARC.

The currently estimated critical baryon and energy densities for forming the quark gluon plasma is about $5 \rho_{0}$ and $2.5 \mathrm{GeV} / \mathrm{fm}^{3}$, respectively [6]. According to the above cascade model prediction, it is highly possible to form the quark-gluon plasma at AGS energies. However, the lifetime and volume of the high density region are significantly reduced by the repulsive mean field. Noticeably, the maximum baryon and energy densities are reduced to about $7 \rho_{0}$ and $2.6 \mathrm{GeV} / \mathrm{fm}^{3}$, respectively, by using the soft nuclear equation of state. With a stiff equation of state corresponding to the compressibility of $K=380 \mathrm{MeV}$ the reduction is even larger although the mean field has almost no effect in the early stage of the reaction when the kinetic energy is much higher than the potential energy. Since the stiff equation of state violates causality already at about $3 \rho_{0}$, we will only use the soft equation of state in the following. The reduction of the maximum baryon and energy densities due to the mean field may be large enough to affect significantly the kind of physical processes that can happen during the reaction.

The validity of any hadronic model for heavy ion collisions at AGS energies is naturally limited by the possible phase transition to the quark-gluon plasma. With this precaution in mind, one can use predictions of hadronic models as a baseline in searching for new phenomena. The formation of the quark-gluon plasma or a mixed phase of hadrons and quark-gluon plasma is expected to reduce the pressure of the system and leads to a softened nuclear equation of state. It is therefore interesting to search for experimental observables that are sensitive to the nuclear equation of state. A careful study on the transverse momentum and rapidity distributions of protons, pions and kaons for the reaction of $\mathrm{Au}+\mathrm{Au}$ at $P_{\text {beam }} / A=11.6 \mathrm{GeV} / \mathrm{c}$ has been done by comparing calculations with and without the mean field. Much to our surprise, both calculations can well reproduce the inclusive, single particle data within current systematic and statistical error bars of the data. The largest difference of about $20 \%$ is found for the proton transverse momentum distribution with a slight shift to higher transverse momenta in the case using the soft equation of state. This 
difference is, however, compatible with the current systematic error bars of the data. These results will be published elsewhere [14].

Hinted by the findings at Bevelac energies that the collective variables or correlation functions, unlike the single particle observables, are very sensitive to the nuclear equation of state, we now turn to the analysis of the baryon transverse collective flow [17]. Here we use the standard flow analysis as in ref. [7]. It was shown recently in ref. [18] that an improved flow analysis with the explicit conservation of reaction plane and angular momentum in individual hadron-hadron collisions results in an increase of the transverse momentum by about $8 \%$ to $23 \%$ at Bevalac energies. This small enhancement will not affect our discussions and conclusions in the following.

We show in Fig. 2 the average transverse momentum of nucleons in the reaction plane as a function of rapidity for the reaction of $\mathrm{Au}+\mathrm{Au}$ at $P_{\text {beam }} / A=11.6 \mathrm{GeV} / \mathrm{c}$ and impact parameters of 2, 6 and $10 \mathrm{fm}$. Significant differences exist between calculations with and without the mean field for the reaction at all of the three impact parameters. In particular, the flow parameter defined as the slope of the transverse momentum distribution at midrapidity is about a factor of 2.5 larger in the case with the mean field. The strength of the so-called "bounce-off" effect at target or projectile rapidities is also much stronger in calculations with the mean field. It is also seen that the collective flow is the strongest in the midcentral collisions.

To see why the collective flow is a sensitive probe of the dynamics in the high density region, we show in Fig. 3 the flow parameter as a function of time for the $\mathrm{Au}+\mathrm{Au}$ reaction at impact parameters of $2 \mathrm{fm}$ and $6 \mathrm{fm}$. It is clear that the flow is mainly generated in the high density region and saturates in the expansion phase. The ratio of final flow parameters in the calculations with and without the mean field is about 2.5 in the reactions at both impact parameters. This difference is large enough for a clear distinction between models when the experimental data is available. For the reaction at an impact parameter of $6 \mathrm{fm}$, the flow parameter undergoes a reduction before saturation. This is due to the reflection of hot baryons from the cold spectator nucleons. It is interesting to note that the decrease 
of pressure in the high density region due to the possible phase transition to guark-gluon plasma is expected to reduce the strength of collective flow. An experimentally measured, significantly smaller flow parameter than the cascade prediction will be a strong indication of the quark gluon plasma formation at AGS energies.

In summary, we have developed a new relativistic transport model for heavy-ion collisions at AGS energies. Within the framework of this model, we have found that the mean field can significantly affect the maximum energy and baryon densities reached in the reaction. The transverse collective flow analysis is suggested as a useful tool to disentangle theoretical models and a possible indicator of the formation of quark-gluon plasma at AGS energies.

We would like to thank many participants to the program "Hot and dense nuclear matter" at the National Institute for Nuclear Theory at Seattle for interesting discussions. In particular, we are indebted to W. Bauer, L.P. Csernai, P. Danielewicz, S. Das Gupta, V. Koch, J. Randrup and Gy. Wolf for their constructive suggestions and criticisms. This research was supported in part by the NSF Grant No. PHY-9212209 and the Welch Foundation Grant No. A-1110. 


\section{REFERENCES}

[1] Proceedings of Heavy Ion Physics at the AGS, HIPAGS'93, 13-15, Jan., 1993, Eds. G.S.F. Stephans, S.G. Steadman, and W.L. Kehoe; Quark Matter 93, Nucl. Phys. A566, 1c (1994).

[2] H. Sorge, H. Stöcker, and W. Greiner, Ann. of Phys. (NY) 192, 266 (1989); R. Mattiello, H. Sorge, H. Stöcker, and W. Greiner, Phys. ReV. Lett. 63, 1459 (1989); H. Sorge, A.V. Keitz, L. Winckelmann, A. Jahns, H. Sorge, H. Stöcker, and W. Greiner, Phys. Lett. B263, 353 (1991).

[3] Y. Pang, T.J. Schlagel, and S.H. Kahana, Phys. Rev. Lett. 68, 2743 (1992); T.J. Schlagel, Y. Pang and S.H. Kahana, Phys. Rev. Lett. 69, 3290 (1992); S.H. Kahana, Y. Pang, T. J. Schlagel and C. Dover, Phys. Rev. C 47, R1356 (1993).

[4] L. Bravina, L.P. Csernai, P. Levai and D. Strottman, Phys. Rev. C 50, 2161 (1994); and references therein.

[5] J.I. Kapusta, A. P. Vischer and R. Venugopalan, Phys. Rev. C 51, 901 (1995).

[6] C.Y. Wong, Introduction to High Energy Heavy-Ion Collisions, (World Scientific, Singapore), 1994.

[7] G.F. Bertsch and S. Das Gupta, Phys. Rep., 160, 189 (1988).

[8] B.A. Li and W. Bauer, Phys. Rev. C 44, 450 (1991); B.A. Li, W. Bauer and G.F. Bertsch, ibid, C 44, 2095 (1991).

[9] Total cross-sections for the reactions of high energy particles, A. Baldini, V. Flaminio, W.G. Moorhead, D.R.O. Morrison, (Springer-Verlag, Berlin), 1988.

[10] B.J. VerWest and R.A. Arndt, Phys. Rev. C 25, 1979 (1980).

[11] Gy. Wolf, W. Cassing and U. Mosel, Nucl. Phys. A552, 549 (1993).

[12] S. Huber and J. Aichelin, Nucl. Phys. A573, 587 (1994). 
[13] Properties and production spectra of elementary particles, A. N. Diddens, H. Pilkuhn and K. Schlüpmann, (Springer-Verlag, Berlin), 1972.

[14] B.A Li, C.M. Ko and G.Q. Li, in preparation.

[15] G.F. Bertsch, M. Gong, L. McLerran, V. Ruuskanen and E. Sarkkinen, Phys. Rev. D 37, 1202 (1988).

[16] C.M. Ko, Z.G. Wu, L.H. Xia and G.E. Brown, Phys. Rev. Lett. 66, 2577 (1991); Phys. Rev. C 43, 1881 (1991).

[17] P. Danielewicz and G. Odyniec, Phys. Lett. B157, 146 (1985).

[18] J. Zhang, S. Das Gupta and C. Gale, Phys. Rev. C50, 1617 (1994).

\section{FIGURE CAPTIONS}

Fig. 1 Evolution of the central local baryon density and energy density in the reaction of $\mathrm{Au}+\mathrm{Au}$ at $P_{\text {beam }} / A=11.6 \mathrm{GeV} / \mathrm{c}$ and $\mathrm{b}=0$.

Fig. 2 Baryon average transverse velocity in the reaction plane as a function of rapidity for $\mathrm{Au}+\mathrm{Au}$ reactions at $P_{\text {beam }} / A=11.6 \mathrm{GeV} / \mathrm{c}$ and three different impact parameters.

Fig. 3 Time evolution of the flow parameter in the reaction of $\mathrm{Au}+\mathrm{Au}$ at $P_{\text {beam }} / A=11.6$ $\mathrm{GeV} / \mathrm{c}$ and the impact parameter of 2 and $6 \mathrm{fm}$. 